\title{
La implementación de los ODS en una asignatura de posgrado: Patrimonio arquitectónico y desarrollo sostenible
}

\section{Andrea Peiró Vitoria ${ }^{a}$ y Laura Gilabert Sansalvador ${ }^{b}$}

${ }^{a}$ ITACA, Instituto Universitario de Tecnologías de la Información y Comunicaciones de la Universitat Politècnica de València (anpeivi@upv.es), ${ }^{b}$ Departamento de Composición Arquitectónica de la Universitat Politècnica de València (laugisan@upv.es).

\begin{abstract}
The University, as an entity that transmits knowledge and transforms society, has the challenge of aligning the curricular program of all the degrees with 2030 Agenda and introducing competencies related to sustainable and inclusive development.

This communication proposes the introduction of SDGs in the subject Sustainable Development and Heritage of the Master's Degree in Architectural Heritage Conservation, which focuses on the design and formulation of management projects linked to architectural heritage. For this purpose, the SDG Compass methodology, especially oriented to the companies, is adapted to incorporate the SDGs in the different project phases. In this way, the aim is to raise awareness and commitment to the Agenda 2030 for Sustainable Development, as well as to enable students to articulate and monitor the Agenda.
\end{abstract}

Keywords: 2030 Agenda, SDGs, architecture, heritage, sustainable development, projects, methodology, skills

\section{Resumen}

La Universidad, como entidad transmisora de conocimiento y transformadora de la sociedad, tiene el reto de alinear el programa curricular de todas las titulaciones con la Agenda 2030 e introducir competencias relacionadas con un desarrollo sostenible e inclusivo.

En la presente comunicación se propone la introducción de los ODS en la asignatura Desarrollo Sostenible y Patrimonio del Máster Universitario en Conservación del Patrimonio Arquitectónico, que se centra en el diseño y formulación de proyectos de gestión vinculados al patrimonio arquitectónico. Para ello, se adapta la metodología SDG Compass, orientada especialmente a empresa, para incorporar los ODS en las diferentes fases proyectuales. De esta forma, se pretende dar a conocer, sensibilizar y comprometer al alumnado con la Agenda 2030 para el Desarrollo Sostenible, a la vez que capacitarle para articular y dar seguimiento a la propia Agenda.

Palabras clave: Agenda 2030, ODS, arquitectura, patrimonio, desarrollo sostenible, proyectos, metodología, competencias 


\section{Introducción}

El 25 de septiembre de 2015, la Asamblea General de las Naciones Unidas aprobó la Agenda 2030 para el Desarrollo Sostenible (ONU, 2015). Esta Agenda, bajo el lema Transformar Nuestro Mundo, constituye un nuevo reto internacional para lograr erradicar la pobreza y favorecer un desarrollo sostenible e igualitario.

La agenda gira entorno a cinco ejes centrales: PLANETA, PERSONAS, PROSPERIDAD, PAZ Y ALIANZAS, disponiendo en el centro de todo, la SOSTENIBILIDAD. Plantea 17 Objetivos de Desarrollo Sostenible (ODS) (Fig. 1) con 169 metas asociadas que definen las prioridades de desarrollo sostenible a nivel mundial abarcando tanto la esfera ambiental, como la económica y la social.

Es una Agenda global e integral que interpela a todos los agentes y actores de la sociedad, y a todos los niveles. Así pues, los ODS implican un compromiso común y universal.

\section{(7) OBJETIVES SEOESARROLO}
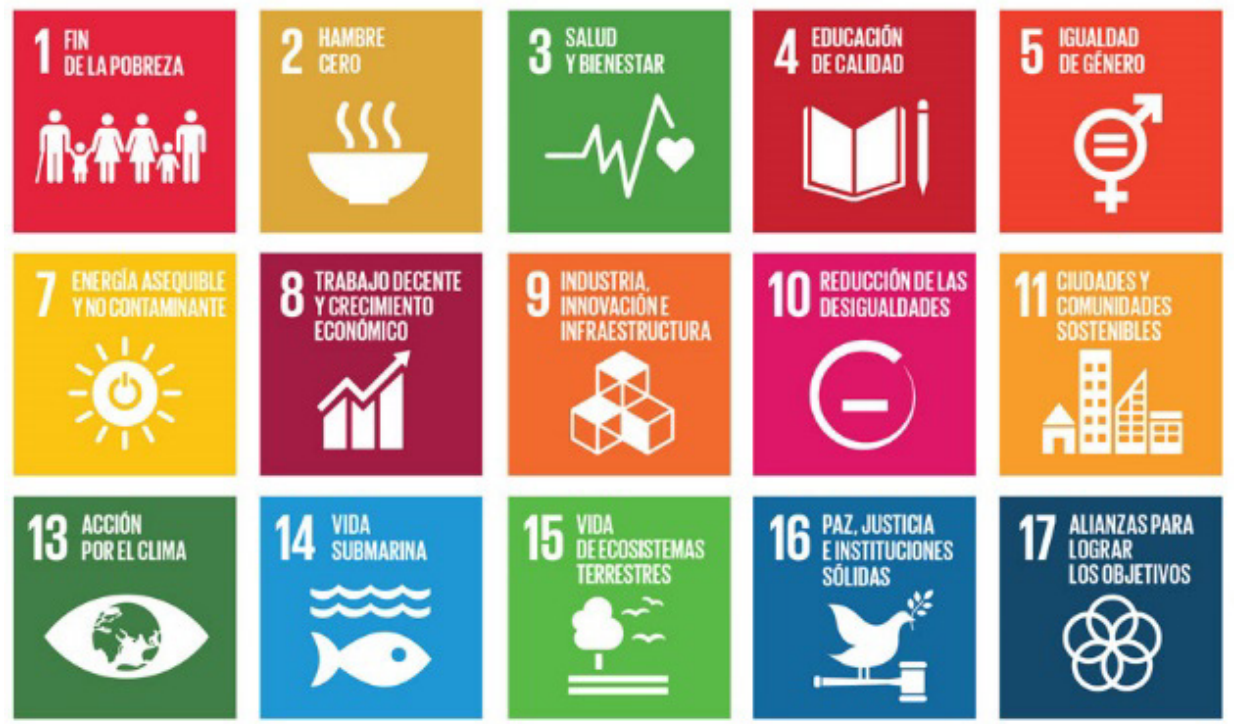
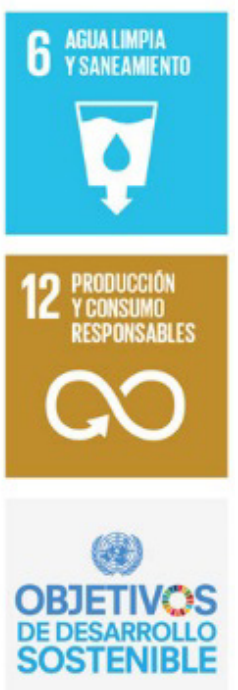

Fig. 1. Los 17 Objetivos de Desarroollo Sostenible de la Agenda 2030 (ONU, 2015).

\subsection{La Universidad y la Agenda 2030}

"La Universidad, dada su naturaleza vinculada a la generación, transferencia y difusión de un conocimiento abierto al servicio de la sociedad, del bienestar y la sostenibilidad, cuenta con las capacidades para realizar una contribución crítica y constructiva al desarrollo sostenible a través de dicho conocimiento" (CRUE, 2018: 1) y el principio de integralidad de la Agenda 2030 interpela a la Universidad de manera directa, como entidad transformadora de la sociedad.

La Agenda contituye una oportunidad, a la vez que un reto. Esta responsabilidad con el desarrollo sostenible, Crue Universidades Españolas ya la asumió en las directrices aprobadas en 2005, y ratificadas en 2011, para incorporar en todas las titulaciones universitarias, competencias en sostenibilidad, entendidas como el "conjunto complejo e integrado de conocimientos, destrezas, habilidades, actitudes y valores que capacitan para operar y transformar la realidad con criterios de sostenibilidad". (CRUE, 2018: 2) 
En mayo de 2018 Crue Universidades Españolas (CRUE, 2018) consensua la aportación conjunta de las universidades al Plan de Acción 2018-2020, impulsado por el Gobierno de España para trabajar en el marco de la Agenda 2030. En este consenso se reflejan, entre otros, los siguientes compromisos:

- Un compromiso decidido con la inclusión de competencias relacionadas con un desarrollo sostenible e inclusivo, necesarias para la construcción de una ciudadanía global, en la formación de todo el estudiantado, el personal docente e investigador y el personal de administración y servicios.

- La generación y la transferencia de un conocimiento comprometido con el desarrollo sostenible, incluyendo aquí también el conocimiento necesario para articular y dar seguimiento a la propia Agenda 2030.

Ambos compromisos adquiridos por la Universidad implican, en el ámbito de la docencia, la incorporación en los programas curriculares tanto de materias y competencias relacionadas con el desarrollo sostenible, como sobre el conocimiento y sistemas de implementación y control de los propios ODS.

Aterrizar los ODS en la educación superior, en aspectos concretos, contextualizados y adaptados a la realidad de cada actor, es complejo. Y a pesar del compromiso de las universidades con la Agenda 2030, la adaptación curricular de las asignaturas y la incorporación de la consecución de los ODS en su programa no es evidente. Aunque en la actualidad se están llevando a cabo un gran número de acciones y actividades relacionadas con el desarrollo sostenible, en la mayoría de los casos, no se encuentran alineadas, de forma consciente, con la Agenda.

En la presente comunicación se expone una propuesta para la introducción de los ODS y la Agenda 2030 en el programa curricular de una asignatura de posgrado que se enfoca en el diseño y la formulación de proyectos vinculados al patrimonio arquitectónico y el desarrollo sostenible.

\subsection{Contextualización de la asignatura de aplicación}

Nos centraremos, concretamente, en la asignatura Desarrollo Sostenible y Patrimonio de la especialidad de Gestión del Máster Universitario en Conservación del Patrimonio Arquitectónico de la Escuela Técnica Superior de Arquitectura de la Universitat Politècnica de València (www.upv.es/titulaciones/MUCPA), cuyo responsable es el Dr. Gaspar Muñoz Cosme, profesor Catedrático del Departamento de Composición Arquitectónica de la UPV. Se trata de una asignatura obligatoria de 4 créditos que se desarrolla en el segundo y último semestre del programa académico. Se imparte desde el curso 2011-2012 y el número medio de alumnos que la cursan es de 12,55. El alumnado es muy diverso, de diferentes titulaciones y nacionalidades. Entre las diferentes titulaciones de las que proviene, arquitectura, historia, historia del arte y restauración de bienes culturales son las más frecuentes. En cuanto a la nacionalidad, en los últimos cursos, hay una mayoría de extranjeros que proviene, sobretodo de países de América Latina, como Colombia, Ecuador, Perú, México, Guatemala o Chile. Aproximadamente el $40 \%$ de los alumnos tienen experiencia en el ámbito del patrimonio o han trabajado en la administración pública de su país de origen, pero en casi ningún caso están familiarizados con el desarrollo sostenible.

El objetivo general de la asignatura es contribuir a la formación integral y competecial del alumno a través del diseño y la formulación de proyectos de desarrollo y patrimonio arquitectónico, aprendiendo a: identificar posibles propuestas, analizar y diagnosticar una situación real y diseñar un proyecto de gestión complejo aplicable a la realidad. Los alumnos llevan a cabo el proceso de identificación, análisis y diseño del proyecto a través de la aplicación de la metodología de Enfoque del Marco Lógico, una herramienta 
analítica ampliamente utilizada en el ámbito de la cooperación al desarrollo (Ferrero y de Loma Osorio, 2010). Es una asignatura de aplicación práctica basada en los principios del desarrollo sostenible y desarrollada a partir de una metodología vinculada al ámbito de la cooperación internacional, lo que la convierte en una candidata ideal para plantear su alineación con la Agenda 2030, mediante la vinculación directa de los proyectos académicos de intervención y gestión del patrimonio con los ODS.

\section{Objetivos y metodología}

Los objetivos generales de la innovación docente propuesta son: informar, sensibilizar y comprometer al alumnado con la Agenda 2030 para el Desarrollo Sostenible.

En cuanto a los objetivos específicos, en correspondencia con las capacidades y conocimientos que el alumnado debe haber adquirido al finalizar el curso vinculados directamente con la innovación docente, son los siguientes:

- Conocer los fundamentos de los Objetivos de Desarrollo Sostenibles y la Agenda 2030.

- Adquirir herramientas analíticas para comprender en profundidad el paradigma del desarrollo sostenible.

- Definir metas y objetivos específicos vinculados con los ODS en el ámbito del patrimonio arquitectónico y establecer estrategias para su implementación.

- Determinar indicadores concretos y medibles que permitar realizan el control y seguimiento futuro de consecución de los ODS en el proyecto propuesto.

En el curso de la asignatura se trabajan varias de las 13 competencias transversales establecidas por la UPV para favorecer la futura inserción del alumnado en el mercado laboral. Con la introducción de los ODS y la Agenda 2030 en el programa curricular de la asignatura se hace hincapié en las sigiuentes:

- CT-02 Aplicación y pensamiento práctico: Encontrar soluciones mediante conocimientos previos, adaptándose a la singularidad de cada situación.

- CT-07 Responsabilidad ética, medioambiental y profesional: Ser conscientes del impacto de nuestras acciones en los demás y en el entorno.

- CT-09 Pensamiento crítico: poner en cuestión las ideas con afán de mejorar.

- CT-10 Conocimiento de problemas contemporáneos: Identificar e interpretar los problemas contemporáneos en su campo de especialización, así como en otros campos del conocimiento, prestando especial atención a los aspectos relacionados con la sostenibilidad.

En cuanto a la metodología para incorporar los ODS en la asignatura, se utilizará el sistema que plantea la Guía SDG Compass (Brújula de los ODS), constituida para orientar a las empresas sobre cómo alinear sus estrategias y medir y gestionar su contribución a los ODS. (GRI, UN Global Compact y WBCSD, 2015: 5)

Esta metodología presenta 5 pasos básicos (Fig. 2):

1. Conocer y entender los ODS.

2. Definir prioridades. Identificar los ODS prioritarios para cada caso.

3. Establecer objetivos específicos y medibles.

4. Integrar. Elaborar estrategias de implementación.

5. Reportar y comunicar los avances. Realizar el seguimiento. 


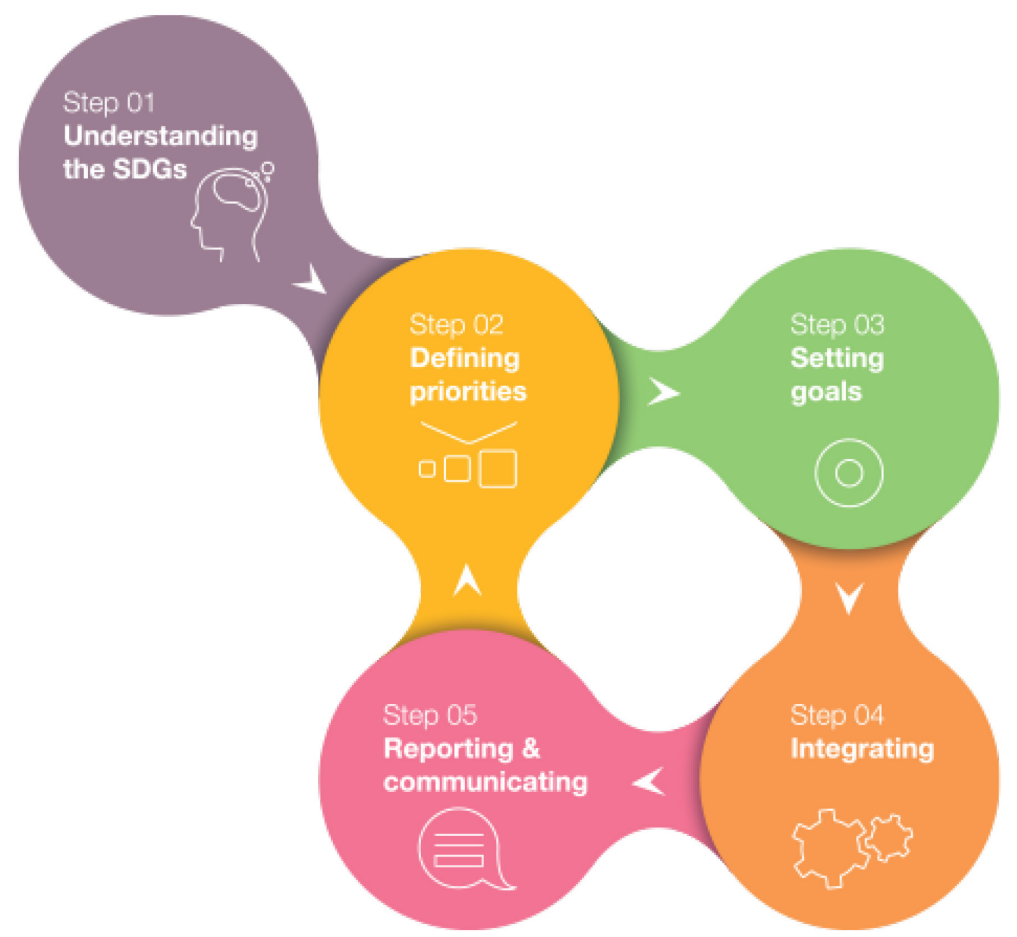

Fig. 2. Los cinco pasos del método SDG Compass (GRI, UN Global Compact y WBCSD, 2015: 5).

Cabe tener en cuenta, que todos los aspectos son importantes, conocer los ODS, saber como articularlos e integrarlos, y también poder dar seguimiento a su implementación mediante indicadores.

\section{Desarrollo de la innovación}

En la asignatura Desarrollo Sostenible y Patrimonio, los alumnos diseñan y formulan un proyecto de gestión de un bien patrimonial arquitectónico a través de la aplicación del Enfoque del Marco Lógico (EML). Esta metodología les permite diagnosticar y analizar situaciones reales complejas y, sobre todo, formular un proyecto de gestión que sea susceptible de recibir financiación pública, por lo que debe contar con mecanismos que faciliten su seguimiento y evaluación.

En la asignatura se combinan sesiones teóricas, en las que se introducen los conceptos del desarrollo sostenible y los principios del método (EML), y sesiones prácticas, en las que se va desarrollando y guiando el proyecto individual que desarrolla cada uno de los alumnos, según esta secuencia:

1. Identificación. En la fase inicial del proyecto los alumnos eligen un bien patrimonial arquitectónico sobre el que actuar. El edificio en cuestión debe tener valores patrimoniales y debe presentar un problema actual evidente, como por ejemplo abandono, mal estado de conservación o desuso. Además de identificar el edificio, deben identificar los beneficiarios de su proyecto, es decir, la comunidad vinculada al bien patrimonial que puede beneficiarse de la recuperación o rehabilitación del edificio, concebida ésta como un motor de desarrollo sostenible para su entorno.

2. Análisis. Una vez identificado el caso de estudio, los alumnos deben realizar un completo análisis de la situación, que incluye: un análisis de los agentes sociales implicados (análisis de participación) y un análisis profundo de los problemas, que estudia las relaciones entre los aspectos negativos de la situación existente, identificando causas y consecuencias. 
3. Objetivos y estrategias. A partir del análisis de los problemas se caracteriza la situación deseable que se quiere alcanzar en el futuro (análisis de objetivos) y los alumnos formulan los objetivos y seleccionan las estrategias de su propuesta.

4. Diseño. En la fase de diseño se concretan las actuaciones y actividades a realizar para alcanzar los objetivos específicos propuestos. Se especifican los medios y los recursos económicos que se necesitan y se realiza una planificación temporal de la ejecución del proyecto.

5. Identificación de instrumentos de evaluación. Finalmente, los alumnos establecen los indicadores que faciliten el seguimiento y la evaluación del proyecto, así como las fuentes de verificación en donde puede encontrarse la información pertinente para verificar el cumplimiento de estos indicadores.

Todo este proceso de formulación del proyecto queda plasmado de forma sintética en la Matriz de Planificación del Proyecto (MPP), una herramienta gráfica que sintetiza todos los aspectos del proyecto y expone los criterios para su seguimiento y evaluación (Sainz Ollero y Gómez Galán, 2003).

La introducción de los ODS se realizará en todas y cada una de las 5 fases del ciclo del proyecto establecidas en correspondencia con los 5 pasos que presenta el método de SDG Compass para la implementación de los ODS en empresas. Y en correlación con la Matriz de Planificación del Proyecto (MPP), se propone la realización de una Matriz de Seguimiento de la Agenda 2030 (MSA) donde se relacione los ODS con las metas y objetivos específicos que se propongan y se expongan los indicadores para realizar su seguimiento.

Como se observa en la figura 3, ambos métodos son perfectamente compatibles y comparten la misma estructura en fases lógicas, pudiendo introducir los ODS a lo largo de todo el proceso proyectual.

\section{Método EML}

1. Identificación

2. Análisis

3. Objetivos y estrategias

4. Diseño

5. Identificación de instrumentos de evaluación

Matriz de Planificación del Proyecto (MPP)

\section{Método SDG Compass (ODS)}

\section{Conocer y entender los ODS}

\section{Definir prioridades. Identificar los ODS} prioritarios para cada caso

\section{Establecer objetivos específicos y medibles vinculados con los ODS}

4. Integrar. Elaborar estrategias de implementación

\author{
5. Reportar y comunicar los avances. Realizar el \\ seguimiento
}

Matriz de Seguimiento de la Agenda 2030 (MSA)

Fig. 3. Tabla de correspondencia entre las metodologías EML y SDG Compass

La Matriz de Seguimiento de la Agenda 2030 (MSA) es la culminación del trabajo de relación del desarrollo del proyecto con los ODS, al igual que la Matriz de Planificación del Proyecto (MPP) es la culminación del EML. En este caso, se trata de una síntesis de la relación de los ODS con los objetivos específicos, metas y criterios de seguimiento y evaluación mediante indicadores. 
Los indicadores son medios, instrumentos o mecanismos para evaluar de forma objetiva hasta qué punto o en qué medida se están logrando unos objetivos preestablecidos, en este caso servirán para dar seguimiento a los objetivos que se establezcan en el proyecto en relación con los ODS dentro del marco de la Agenda 2030. Para poder trabajar con ello, el alumnado deberá conocer los documentos básicos de partida, como el listado de indicadores desarrollado por el Grupo Interinstitucional de Expertos sobre indicadores ODS (IAEG-ODS) de Naciones Unidas o los indicadores considerados por Sustainable Development Solutions Network, SDSN (2015), además de los indicadores establecidos en el Marco de indicadores mundiales para los Objetivos de Desarrollo Sostenible y metas de la Agenda 2030 para el Desarrollo Sostenible (A/RES/71/313). Es importante tener en cuenta que, al igual que la Agenda 2030 no es un proceso cerrado, las tablas de indicadores también deben ser dinámicas y adaptables a los cambios de situación, contexto y retos de cada caso particular (Gómez Torres et al. 2019).

La Matriz de Seguimiento de la Agenda 2030 (Fig. 4) incluirá los siguientes apartados, atendiendo a las diferentes fases de desarrollo del proyecto:

- ODS de la Agenda 2030 con el que se vincula

- Objetivo específico concreto adaptado al caso específico del proyecto

- Meta que se pretende conseguir con esa acción

- Indicador específico y medible para dar seguimiento a la consecución de ese objetivo

- Fuente de verificación, el cómo y dónde se puede conseguir el dato objetivo para realizar ese seguimiento

- Justificación de la elección de ese objetivo o hipótesis de partida que demuestra la necesidad de alcanzar esa meta y que garantice su viabilidad

\begin{tabular}{|l|l|l|l|l|l|}
\hline ODS & $\begin{array}{l}\text { Objetivo } \\
\text { específico }\end{array}$ & Meta & Indicador & $\begin{array}{l}\text { Fuente de } \\
\text { verificación }\end{array}$ & $\begin{array}{l}\text { Justificación o } \\
\text { hipótesis de partida }\end{array}$ \\
\hline & & & & & \\
\hline & & & & & \\
\hline & & & & & \\
\hline & & & & & \\
\hline
\end{tabular}

Fig. 4. Matriz de Seguimiento de la Agenda 2030 (MSA) que deberán cumplimentar

\subsection{Planificación de actividades vinculadas a los objetivos específicos}

Las diferentes fases de la metodología propuesta de implementación de los ODS de la Agenda 2030 en el desarrollo de proyectos (método SDG Compass) se relacionan con los 4 objetivos específicos de la siguiente forma (Fig.5). 


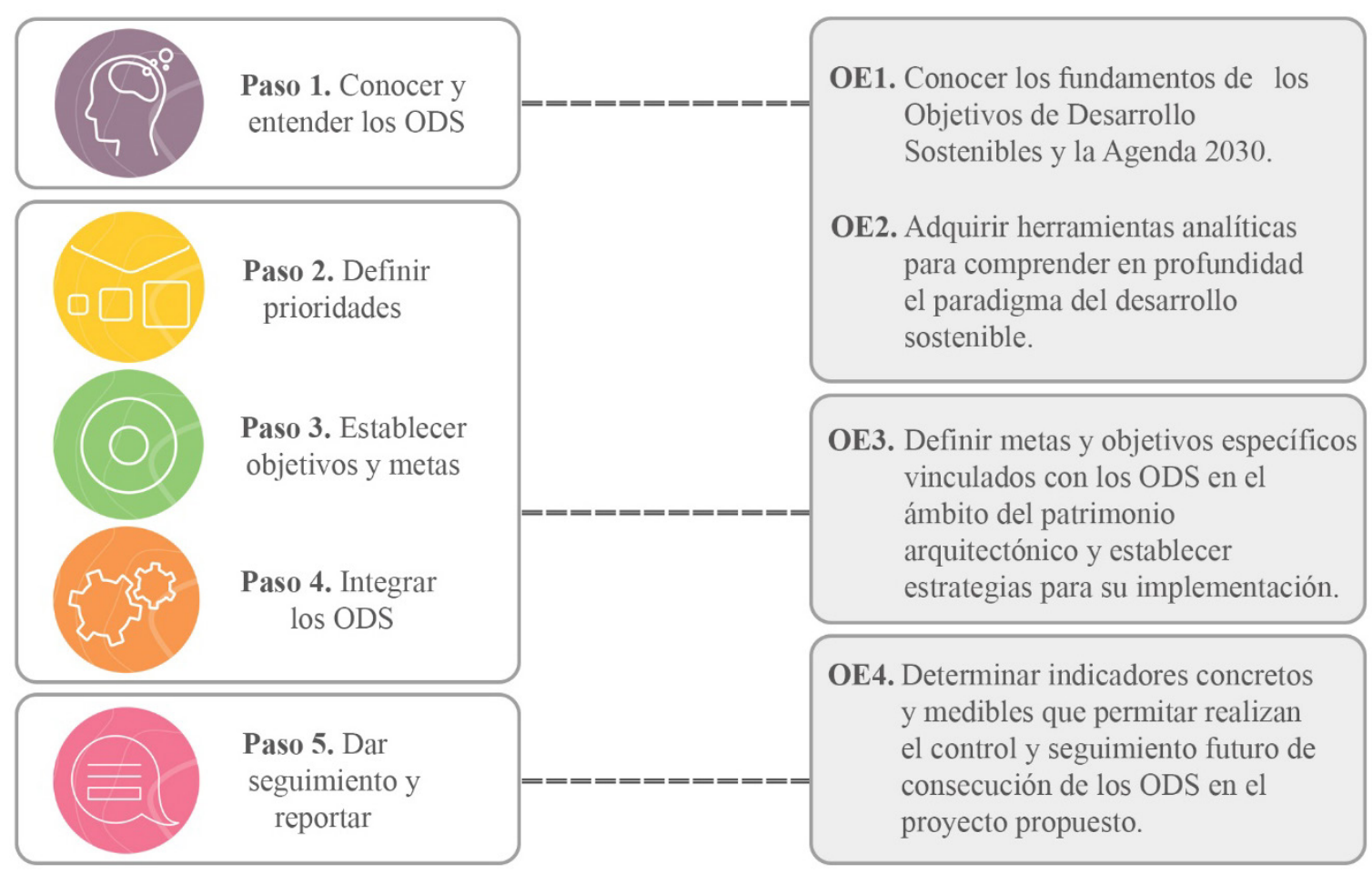

Fig. 5. Relación de los pasos del Método SDG Compass con los Objetivos Especificos.

Como se puede observar, el orden de los objetivos específicos se corresponde con el nivel de profundización en el conocimiento y con el avance en el desarrollo del proyecto individual que el alumnado debe realizar a lo largo del curso, al igual que los 5 pasos de la metodología propuesta.

Para conseguir alcanzar estos objetivos específicos, se plantea una serie de actividades.

\section{OE1 Conocer los fundamentos de los Objetivos de Desarrollo Sostenibles y la Agenda 2030}

1. Clase introductoria con el método Flip Teaching

Se entregará a los alumnos un texto y un vídeo sobre la Agenda 2030 y los ODS para que lo lean y estudien en casa anteriormente a la clase.

Después en clase se realizará un trabajo por grupos en el que pondrán en común la información adquirida en casa.

Por último, comentarán y debatirán con el resto de la clase cómo consideran que se puede aplicar los ODS a la práctica profesional en el ámbito del patrimonio arquitectónico.

2. Conferencia invitada

Se invitará a un profesional especializado en los ODS para explicar la Agenda 2030 y los ODS, exponiendo tanto el punto de partida, como el procedimiento seguido por la ONU para la concreción de los ODS y los reusltados esperados, mediante las metas definidas.

Resultado esperado:

Que los alumnos se familiaricen con la Agenda 2030, que sepan reconocer los 17 ODS y que acepten el reto que supone para todos y cada uno de los agentes de esta sociedad. 


\section{Evidencias:}

Tras finalizar esta serie de actividades, cada alumno responderá a un cuestionario con diversas preguntas cortas: ¿qué son los ODS? ¿de dónde viene la Agenda 2030? ¿a qué retos nos enfrentamos en esta nueva era? ¿cómo consideras que pueden aterrizarse los ODS en el ámbito del patrimonio arquitectónico? Pon un ejemplo de aplicación.

OE2 Adquirir herramientas analíticas para comprender en profundidad el paradigma del desarrollo sostenible

1. Workshop con uno o varios especialistas en la Agenda 2030 como profesores invitados Se invitará a varios profesionales del ámbito de la cooperación y especialistas en ODS, como por ejemplo, algún miembro de la Comisión Sectorial CRUE-Sostenibilidad, para plantear y analizar con los alumnos algunos de los retos a los que se enfrenta hoy en día la sociedad.

2. Debates en clase siguiendo el método Flip Teaching

Se entregará a los alumnos diferentes casos de intervención y gestión de patrimonio arquitectónico que deberán leer y estudiar en casa, previo a la clase.

Posteriormente, en clase, debatirán en grupos: analizarán los diferentes casos, relacionándolos con los ODS y plantearán acciones concretas vinculadas a los mismos que se podrían implementar en un futuro.

Resultado esperado:

Que el alumnado se haya adentrado con mayor profundidad en el conocimiento de los ODS, con una capacidad crítica y que puedan aplicar ese conocimiento general en casos prácticos de carácter local.

\section{Evidencias:}

Informe que sintetice las conclusiones del debate en grupo: información del caso, vínculos con los ODS y posibles acciones a implementar.

OE3 Definir metas y objetivos específicos vinculados con los ODS en el ámbito del patrimonio arquitectónico y establecer estrategias para su implementación

1. Clase-Taller: Introducción de los ODS en el proceso de desarrollo individual del proyecto de gestión de patrimonio arquitectónico

En las clases prácticas de desarrollo del proyecto, paralelamente a la realización de las fases 2, 3 y 4 de análisis, de definición de objetivos y estrategias, y de diseño del proyecto, se estudiarán y definirán las prioridades concretas y metas a alcanzar vinculadas a los ODS (pasos 2 y 3 del métodos SDG Compass) y se integrarán en el proyecto en todas y cada una de sus etapas de implementación (paso 4 SDG Compass).

\section{Resultado esperado:}

Que el alumno analice los riesgos y oportunidades de un caso real, que aprenda a identificar los posibles ámbitos de actuación en su proyecto en vinculación con los ODS y que pueda concretar las metas y objetivos específicos extrapolando de lo global a lo local.

\section{Evidencias:}

El proyecto final entregado. 
OE4 Determinar indicadores concretos y medibles que permitar realizan el control y seguimiento futuro de consecución de los ODS en el proyecto propuesto

1. Clase introductoria con el método Flip Teaching

Se entregará a los alumnos varios textos sobre el sistema de medición por indicadores, procesos de seguimiento y evaluación de los ODS en el marco de la Agenda 2030 para que los lean en casa.

En clase se comentarán los textos y se pondrá en práctica la definición de algunos indicadores en casos cercanos propuestos por el profesor.

2. Clase-Taller: Definición de indicadores y fuentes de verificación específicas sobre la incorporación de los ODS en el proyecto de gestión de patrimonio arquitectónico En las clases prácticas de desarrollo del proyecto, el alumnado deberá determinar qué indicadores serán de utilidad para dar seguimiento a los objetivos específicos propuestos en el proyecto, indicando las fuentes de verificación.

Resultado esperado:

Que el alumnado sepa aplicar un método de seguimiento y evaluación de objetivos específicos de un proyecto vinculados con los ODS de la Agenda 2030.

\section{Evidencias:}

La Matriz de Seguimiento de la Agenda 2030 (MSA), paralelamente a la Matriz de Planificación de Proyecto (MPP).

\subsection{Evaluación}

Por último, para evaluar si se han alcanzado los objetivos específicos, se aplicará la siguiente rúbrica de evaluación (Fig. 6) en correspondencia con las evidencias de las actividades propuestas:

\begin{tabular}{|c|c|c|c|}
\hline & INSUFICIENTE & BIEN & EXCELENTE \\
\hline $\begin{array}{l}1 \text { Cuestionario sobre } \\
\text { el conocimiento de los } \\
\text { ODS (OE1) }\end{array}$ & $\begin{array}{l}\text { No ha comprendido el } \\
\text { alcance de la Agenda } 2030 \\
\text { ni los ODS. No encuentra } \\
\text { aplicación al ámbito del } \\
\text { patrimonio arquitectónico. }\end{array}$ & $\begin{array}{l}\text { Ha adquirido un } \\
\text { conocimiento básico sobre } \\
\text { la Agenda } 2030 \text { y los } \\
\text { ODS, pero no ha } \\
\text { alcanzado a comprender } \\
\text { su vinculación con el } \\
\text { patrimonio arquitectónico. }\end{array}$ & $\begin{array}{l}\text { Demuestra un conocimiento } \\
\text { amplio sobre la Agenda } \\
2030 \text { y los ODS y sabe } \\
\text { enlazarlos fácilmente con el } \\
\text { ámbito del patrimonio } \\
\text { arquitectónico. }\end{array}$ \\
\hline $\begin{array}{l}2 \text { Informe del debate } \\
\text { en grupo: } \\
\text { información del caso, } \\
\text { vínculos con los ODS } \\
\text { y posibles acciones a } \\
\text { implementar (OE2) }\end{array}$ & $\begin{array}{c}\text { No demuestra la } \\
\text { vinculación del caso de } \\
\text { estudio con ningún ODS. }\end{array}$ & $\begin{array}{l}\text { Demuestra la vinculación } \\
\text { del caso de estudio con los } \\
\text { ODS, pero no concreta } \\
\text { correctamente las acciones } \\
\text { a implementar. }\end{array}$ & $\begin{array}{l}\text { Demuestra perfectamente } \\
\text { la vinculación del caso de } \\
\text { estudio con los ODS y } \\
\text { define correctamente las } \\
\text { acciones a implementar. }\end{array}$ \\
\hline $\begin{array}{c}3 \text { Estudio de } \\
\text { prioridades y } \\
\text { selección de los ODS } \\
\text { vinculados con el } \\
\text { proyecto (OE3) }\end{array}$ & $\begin{array}{l}\text { Los ODS seleccionados no } \\
\text { se alinean con las } \\
\text { prioridades detectadas y la } \\
\text { vinculación de los ODS } \\
\text { con el proyecto no queda } \\
\text { claramente justificada. }\end{array}$ & $\begin{array}{l}\text { Los ODS seleccionados se } \\
\text { alinean con las prioridades } \\
\text { detectadas, pero la } \\
\text { vinculación de los ODS } \\
\text { con el proyecto no queda } \\
\text { claramente justificada. }\end{array}$ & $\begin{array}{l}\text { Los ODS seleccionados se } \\
\text { alinean perfectamente con } \\
\text { las prioridades detectadas y } \\
\text { la vinculación de los ODS } \\
\text { con el proyecto queda } \\
\text { claramente justificada. }\end{array}$ \\
\hline
\end{tabular}




\begin{tabular}{|c|c|c|c|}
\hline $\begin{array}{l}4 \text { Definición de metas } \\
\text { y objetivos específicos } \\
\text { del proyecto (OE3) }\end{array}$ & $\begin{array}{l}\text { Las metas y objetivos } \\
\text { específicos no están bien } \\
\text { definidos, no se centran en } \\
\text { las necesidades del } \\
\text { proyecto y no se concretan } \\
\text { lo suficiente. }\end{array}$ & $\begin{array}{l}\text { Las metas y objetivos } \\
\text { específicos están bien } \\
\text { definidos y se centran en } \\
\text { las necesidades del } \\
\text { proyecto, pero no se } \\
\text { concretan lo suficiente. }\end{array}$ & $\begin{array}{l}\text { Las metas y objetivos } \\
\text { específicos están bien } \\
\text { definidos, se centran en las } \\
\text { necesidades del proyecto y } \\
\text { se concretan lo suficiente } \\
\text { adaptándose al contexto. }\end{array}$ \\
\hline $\begin{array}{c}5 \text { MSA Matriz de } \\
\text { Seguimiento de la } \\
\text { Agenda } 2030 \text { (OE4) }\end{array}$ & $\begin{array}{l}\text { No relaciona de forma } \\
\text { correcta todos los } \\
\text { conceptos. Los objetivos, } \\
\text { los indicadores y las } \\
\text { fuentes de verificación no } \\
\text { están bien relacionados } \\
\text { entre sí, ni están bien } \\
\text { definidos. }\end{array}$ & $\begin{array}{c}\text { Relaciona de forma } \\
\text { correcta los diferentes } \\
\text { conceptos. Los objetivos } \\
\text { están bien relacionados y } \\
\text { definidos, no así los } \\
\text { indicadores y las fuentes } \\
\text { de verificación. }\end{array}$ & $\begin{array}{l}\text { Relaciona perfectamente los } \\
\text { diferentes conceptos. Tanto } \\
\text { los objetivos como los } \\
\text { indicadores y las fuentes de } \\
\text { verificación se relacionan } \\
\text { de forma correcta y están } \\
\text { bien definidos. }\end{array}$ \\
\hline
\end{tabular}

Fig. 6. Rúbrica de evaluación

\section{Resultados}

Los resultados que cabe esperar de la implementación de esta propuesta son, por un lado que el alumnado conozca la Agenda 2030, los ODS en el marco de la misma y el reto global que supone para todos y cada uno de los actores que intervienen en la sociedad. Y por otro, que el alumnado se comprometa con la Agenda 2030 y esté capacitado para analizar, implementar y dar seguimiento a los ODS en su ámbito disciplinario, de forma que este aprendizaje le sirva para la aplicación futura de estos conocimientos en su vida profesional, transfiriéndose así un conocimiento comprometido con el desarrollo sostenible.

En este último curso 2019/20 la Universitat Politécnica de Valencia, a través de la convocatoria de Proyectos de Mejora e Innovación Docente (PIME), ha promovido la introducción de los ODS en las distintas titulaciones, utilizando como punta de lanza el Trabajo Final de Grado (TFG) o de Máster (TFM). La Escuela Técnica de Arquitectura Superior, consciente del interés de esa propuesta y de que la arquitectura como servicio a la sociedad ya atiende a varios de estos objetivos de desarrollo sostenible, planteó introducir esta iniciativa, solicitando como anexo al TFG un informe que indicara la relación del tema del trabajo con los ODS, desde la fase inicial de ideación, de tal forma que se tome conciencia de los ODS desde el principio.

En el caso de los estudios de un máster de especialización como el Máster en Conservación del Patrimonio Arquitectónico, la introducción de los ODS en una asignatura del programa curricular ayudará a los alumnos y les ofrecerá herramientas para implementarlos también en su TFM, afianzando así los conocimientos y capacidades adquiridas. Además, estará capacitado para participar en el concurso que la UPV ha convocado por segundo año ya, con lema "La UPV con los Objetivos de Desarrollo Sostenible. II Concurso de Trabajos Fin de Grado y Tesinas Fin de Master en pro del Desarrollo Humano Sostenible" (Resolución del 15/01/2020 del Rector de le UPV), financiado por la Conselleria de Participació, Transparencia, Cooperació i Qualitat Democràtica de la Generalitat Valenciana. A través de este concruso, se premia el compromiso con los ODS de los TFG y TFM en el avance de la Agenda 2030 para el Desarrollo Sostenible, reconociendo así la participación de la comunidad universitaria en los ámbitos de la solidaridad, sostenibilidad y cooperación al desarrollo.

El éxito de la primera convocatoria de este concurso, en la que se otorgaron dos primeros premios de la modalidad TFG, uno en la categoría de TFM y dos accésits, uno por cada modalidad, muestra los buenos 
resultados al implicar al alumnado en la implementación de los ODS en sus proyectos finales e incentivar mediante un concurso el desarrollo de los mismos.

Con ello, cabe esperar que la implementación de los ODS en la asignatura Desarrollo Sostenible y Patrimonio del Máster Universitario en Conservación del Patrimonio Arquitectónico, obtenga muy buenos resultados, con un alumnado comprometido con la Agenda 2030, con recursos y capacidades para extrapolar ese concimiento a la vida profesional, tanto en el ámbito nacional como internacional, y que además, colabore y potencie la iniciativa general lanzada por la UPV para introducir los ODS en todas las titulaciones.

\section{Conclusiones}

La implementación efectiva de los ODS en los programas curriculares de asignaturas ya consolidadas supone un reto y una dificultad, pero la Universidad, como agente transformador de la sociedad, tiene la obligación y el compromiso adquirido de incluir en la formación competencias relacionadas con un desarrollo sostenible e inclusivo, necesarias para la construcción de una ciudadanía global.

La introducción de los ODS en la asignatura Desarrollo Sostenible y Patrimonio del Máster Universitario en Conservación del Patrimonio Arquitectónico implica la ampliación de los objetivos tanto generales como específicos de la asignatura, dando cabida a aspectos referidos específicamente a la Agenda 2030. Además, conlleva definir una metodología que encaje con la asignatura para poder introducir los ODS desde el principio, pasando por las diferrentes fases: conocimiento, asimilación de conceptos y adquisición de capacidades para evaluar y dar seguimiento.

En el contexto de la asignatura citada, se propone utilizar la metodología SDG Compass que consta de 5 pasos (Conocer y entender, definir prioridades, establecer objetivos específicos, integrar, dar seguimiento y reportar) y que enlaza perfectamente con el método EML que se está aplicando en la actualidad, consiguiendo así introducir los ODS en todas las etapas de diseño del proyecto, desde su ideación inicial hasta el desarrollo final. La propuesta incluye la planificación de diferentes actividades durante todo el curso, cada una de ellas con evidencias para poder comprobar el cumplimiento de los resultados de aprendizaje esperados, y una metodología de evaluación alineada con los objetivos específicos formulados para el aprendizaje de los ODS.

Con la inclusión de los ODS en la asignatura se consigue transferir un conocimiento comprometido con el desarrollo sostenible y capacitar al alumnado para implementar, articular y dar seguimiento a la Agenda 2030. El alumnado, comprometido con los ODS, podrá aplicar ese conocimiento y capacidades en su ámbito profesional, colaborando así a la mejora global y aceptando el reto internacional de conseguir un desarrollo sostenible e igualitario de todos y para todos.

\section{Referencias}

CRUE (2018). El compromiso de las universidad españolas con la Agenda 2030.

FERRERO Y DE LOMA OSORIO, G. (2010). Identificación y formulación de proyectos de cooperación para el desarrollo: gestión del ciclo del proyecto y Enfoque del Marco Lógico. Valencia: Editorial Universitat Politècnica de València.

GÓMEZ TORRES, M. Ll.; PEIRÓ VITORIA, A.; FERNÁNDEZ-BALDOR MARTÍNEZ, Á.; GÓMEZ GÓMEZ, D.; PÉREZ MEDINA, S. y PUCHADES PLA, R. (2019). Elaboración de indicadores para el seguimiento de la Agenda 2030 en la Universidad Española. Comunicación oral en VIII Congreso Universidad y Cooperación al 
Desarrollo. Conocimiento y compromiso social ante los retos globales. 27-29 noviembre 2019. Santiago de Compostela: Observatorio OCUD.

GRI, UN Global Compact y WBCSD (2015). SDG Compass. La guía para la acción empresarial en los ODS.

ONU (2015). Transformar nuestro mundo: la Agenda 2030 para el Desarrollo Sostenible. Resolución aprobada por la Asamblea General de 25 de septiembre de 2015.

SAINZ OLLERO, H. y GÓMEZ GALÁN, M. (2003). El ciclo del proyecto de cooperación al desarrollo. La aplicación del marco lógico. Madrid: CIDEAL.

SDSN (2015). Indicators and a Monitoring Framework for Sustainable Development Goals: Launching revolution for the SDGs. New York \& Paris: Sustainable Development Solutions Network. 